Trauma Berufskrankh 2004 - 6 [Suppl 4] : S455-S463 DOI 10.1007/s10039-004-0845-1

Online publiziert: 20. April 2004

(C) Springer-Verlag 2004

N.P.Südkamp · W. Köstler

Department Orthopädie und Traumatologie, Klinik für Traumatologie, Universitätsklinikum Freiburg

\title{
Minimalinvasive Unfallchirurgie
}

reich der Knieregion werden aus der Vielzahl der Möglichkeiten ausgewählte Techniken dargestellt.

\section{Ellenbogengelenk}

Die Arthroskopie des Ellenbogens wird im Vergleich zu anderen großen Gelenken weniger häufig durchgeführt. Bei frischen Ellenbogenluxationen wird sie eingesetzt, um das als Folge der Verletzung entstandene Hämarthros auszuwaschen und damit die Inzidenz von periartikulären Ossifikationen zu vermindern. Die häufigeren Indikationen zur Ellenbogenarthroskopie sind Folgezustände nach Verletzungen und Erkrankungen. Hier steht an erster Stelle die Entfernung freier Gelenkkörper, die nach Verletzungen oder Erkrankungen (Osteochondrosis dissecans) auftreten können, und die arthroskopische Arthrolyse für die Behandlung von verbliebenen Bewegungseinschränkungen nach vorausgegangenen Verletzungen und Operationen.

\section{Oberes Sprunggelenk}

Arthroskopische und arthroskopisch assistierte Operationen sind deutlich häufiger als am Ellenbogen. Die Indikationen sind ganz ähnlich wie an diesem und beinhalten im Wesentlichen nur Folgezustände nach Verletzungen.

Häufiger als freie Gelenkkörper findet sich eine Osteochondrosis dissecans. Hier kann unter arthroskopischer und röntgenologischer Kontrolle mittels Bildverstärker durch eine Anbohrung des Osteochondroseherds im frühen Stadium I eine
Ausheilung der Erkrankung bewirkt werden.

Als Folge von repetitiven Sportverletzungen, besonders bei Fußballspielern, aber auch nach Frakturen, findet man häufig eine Bewegungseinschränkung bezüglich der Dorsalflexion des Fußes. Diese ist überwiegend Folge eines tibio-talaren Impingements, das durch osteophytäre Knochenanbauten an der Tibiavorderkante oder im tibialen Recessus des Talushalses entsteht. Hier ist die arthroskopisch und radialogisch (Bildverstärker) kontrollierte Abtragung mit arthroskopischen Instrumenten eine exzellente, effektive und komplikationsarme Technik.

Bei ausgeprägten Arthrosen des oberen Sprunggelenks ist in Abhängigkeit von der Beschwerdesymptomatik oft nur noch eine Arthrodese Therapie der Wahl. Sie kann bei erhaltener Kongruenz und fehlender massiver Deformität von distaler Tibia und Talus arthroskopisch minimalinvasiv durchgeführt werden. Um die arthroskopische Bearbeitung der ehemaligen Gelenkflächen zu erleichtern, wird das Gelenk temporär mit einem Fixateur externe distrahiert. Anschließend wird unter bildwandlergestützter Kontrolle perkutan eine Schraubenarthrodese durchgeführt. Hierzu werden unter Bildwandlerkontrolle Kirschner-Drähte eingebracht, über die kanülierte Schrauben eingedreht werden können (• Abb. 1). So gelingt über kleine Stichinzisionen ohne weitere Eröffnung eine Versteifung des aufgebrauchten Gelenks. Aufgrund guter klinischer Erfahrungen hat sich diese Technik bereits vielfach durchgesetzt. 


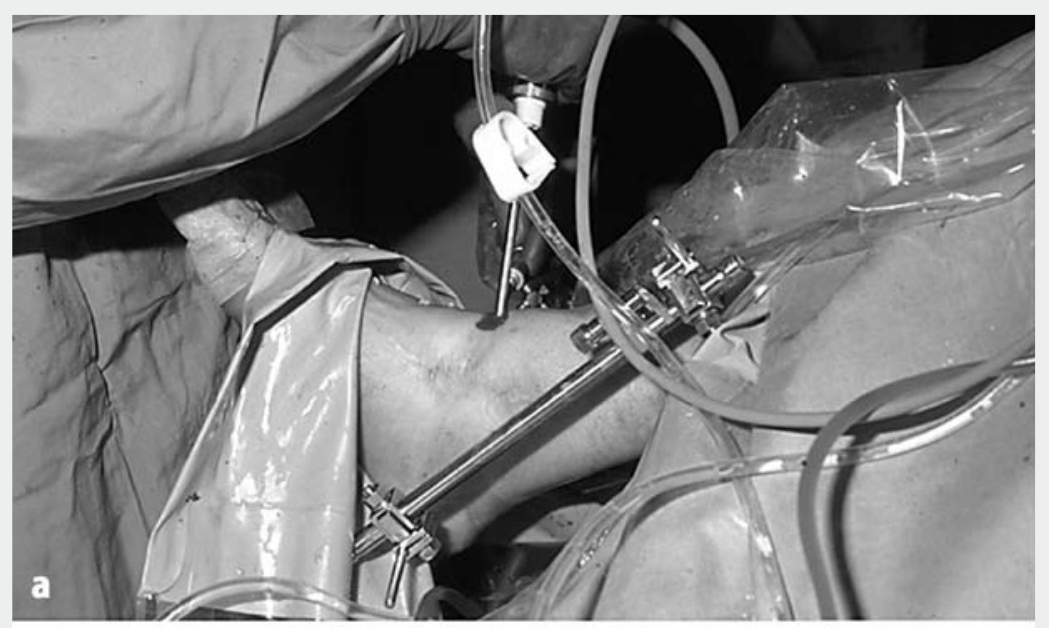

Abb. 1a-c $<$ Technik der Sprunggelenkarthrodese: a arthroskopische Bearbeitung der Gelenkflächen des mit einem Fixateur externe temporär distrahierten Gelenks, b perkutane Arthrodese durch bildwandlergestützte Positionierung von Drähten für die spätere Einbringung von kanülierten Schrauben, c postoperatives Röntgenbild in 2 Ebenen
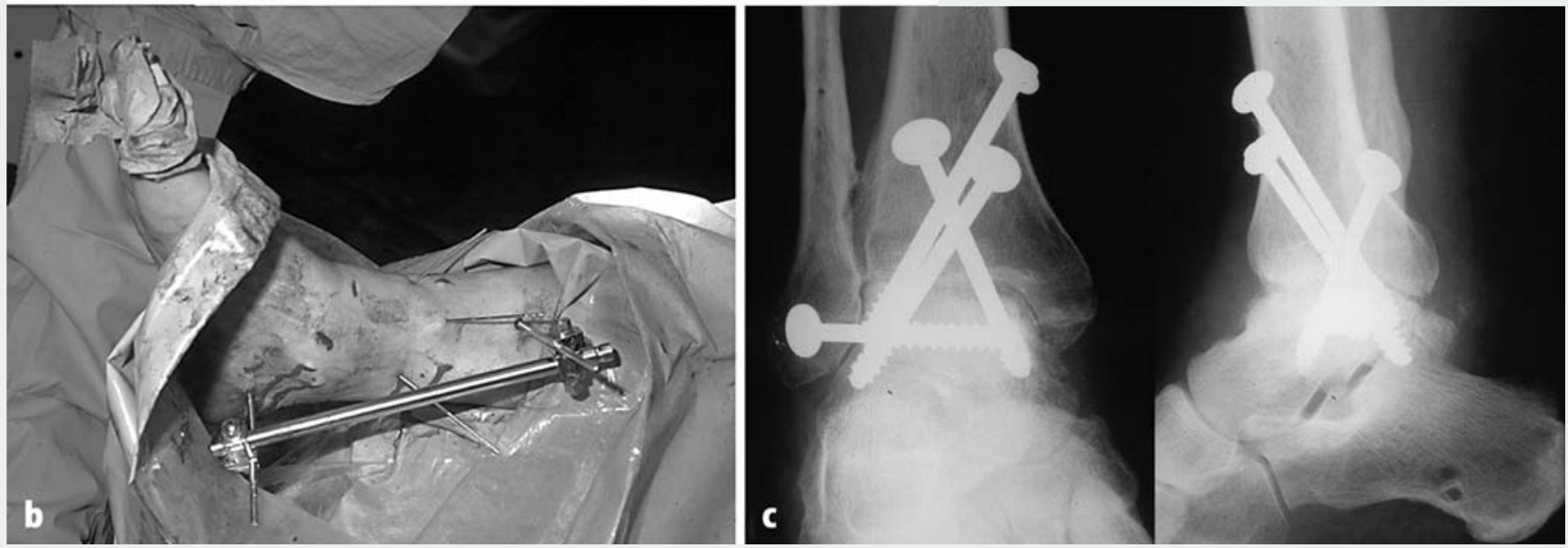

Weitere minimalinvasive Techniken im Sprunggelenkbereich sind die perkutanen Plattenosteosynthesen zur Behandlung von Frakturen der distalen Tibiametaphyse und von Pilon-tibiale-Frakturen. Diese minimalinvasiven Plattenosteosynthesen (MIPO) und minimalinvasiven perkutanen Plattenosteosynthesen (MIPPO) wurden entwickelt, um das operative Trauma (Freilegung des Operationsgebiets) zu minimieren und eine weitere, operativ bedingte Störung der Durchblutung der bereits durch die Fraktur geschädigten Knochenregion zu verhindern (• Abb. 2).

\section{Schultergelenk}

Es ist das Gelenk mit den zweithäufigsten Indikationen für arthroskopische Operationen. Heutzutage können alle Formen der akuten und chronischen Instabilitäten arthroskopisch versorgt werden, zunehmend auch Risse und Defekte der Rotatorenmanschette. Von den degenerati- ven Erkrankungen können neben dem Impingementsyndrom auch die Tendinitis calcarea und die AC-Gelenkarthrose erfolgreich arthroskopisch und damit minimalinvasiv, d. h. Kosten sparend durch z. B. ambulante Operation, kosmetisch ansprechend durch nur kleine Stichinzisionen und komplikationsarm bzw. komplikationslos behandelt werden.

Im Bereich des Schultergelenks sind die Schwerpunkte für arthroskopische Operationen

- Instabilitäten

- Impingementsyndrome

- Tendinitis calcarea

- AC-Gelenkerkrankungen und -verletzungen

- Rupturen der Rotatorenmanschette

\section{Schulterinstabilität}

Die Schulter weist gegenüber anderen Gelenken ein großes Bewegungsausmaß, da- für aber eine vergleichsweise schlechte intrinsische Stabilität auf. Dadurch ist sie leichter verletzbar als andere Gelenke bzw. ist die Luxationsrate wesentlich höher als bei anderen großen Gelenken.

Eine Schulterluxation ist bei eigentlich allen Sportarten möglich, wenn bestimmte Krafteinwirkungen und Gelenkstellungen vorliegen, es gibt jedoch eine Reihe von Sportarten, bei denen sie eine sportartspezifische Verletzung darstellt: Die wichtigsten davon sind Handball, alpiner Skilauf, Eishockey, Rugby, Ringen, Judo, Karate, Geräteturnen, Leichtathletik. Häufig ist bei Sportlern auch mit chronischen Überlastungen zu rechnen, die zwar keine Schulterluxation nach sich ziehen, aber zu Subluxationsphänomenen mit deutlicher klinischer Symptomatologie führen. Auch findet man bei Sportlern mit der gleichen Häufigkeit wie in der normalen Population anlagebedingte bzw. kongenitale Veränderungen, die bei bereits geringer Krafteinwirkung zu rezidi- 
vierenden Schulterverrenkungen führen können.

Die reine traumatische vordere Schulterluxation, bei der eine entsprechende Gewalt auf das Schultergelenk einwirkt, ist die wohl am besten beschriebene Instabilitätsform. Bereits 1890 wiesen Broca u. Hartmann auf die resultierende Läsion im vorderen unteren Bereich des Pfannenrands hin. Bankart erkannte in dieser Läsion die Hauptursache für das erneute Auftreten einer Luxation. Entsprechend den Untersuchungen von Reeves [26] kommt es bei einer traumatischen Schulterluxation beim jüngeren Patienten überwiegend zu einem Abriss des Labrums am vorderen Glenoid, während beim älteren Menschen lediglich die Kapsel oder die Rotatorenmanschette reißen. Verschiedene Autoren beschrieben entsprechend hohe altersabhängige Reluxationsraten nach adäquatem Trauma von über $90 \%$. Bei Patienten, die älter als 45 Jahre sind, kommt es eher zu Verletzungen der Rotatorenmanschette, Reluxationen sind die Ausnahme.

Die von Thomas u. Matsen [37] geprägten Akronyme TUBS und AMBRI repräsentieren die gesamte Bandbreite der möglichen Schulterinstabilitäten. TUBS steht für traumatische Instabilität, $u$ nidirektional, Bankart-Läsion und gute chirurgische Behandelbarkeit (surgery). Der Gegensatz hierzu, AMBRI, ist die Instabilität mit atraumatischer Ätiologie, multidirektional, bilateralen Veränderungen und gutem Ansprechen auf Rehabilitation. Schneeberger et al. [27] haben 1997 eine inzwischen weit akzeptierte Klassifikation aufgestellt, die heute am ehesten gebräuchlich ist (• Tabelle 1).

\section{Arthroskopische Therapie}

Die traumatische vordere Schulterinstabilität eignet sich hervorragend für eine arthroskopische Operation. Es gibt inzwischen zahlreiche Techniken, die alle das gleiche Operationsprinzip aufweisen, nämlich eine anatomische Refixation des abgerissenen Labrums am vorderen Glenoidrand, lediglich die Fixationsmethoden differieren.

Wir verwenden für die operative Versorgung der frischen oder rezidivierenden traumatischen vorderen Schulterluxationen das Verfahren nach Caspari u.
Trauma Berufskrankh 2004 - 6 [Suppl 4] : S455-S463

DOI 10.1007/s10039-004-0845-1

C Springer-Verlag 2004

\section{N.P.Südkamp ·W. Köstler}

\section{Minimalinvasive Unfallchirurgie}

\section{Zusammenfassung}

Ein wesentlicher Bestandteil „, minimalinvasiver Unfallchirurgie" sind die arthroskopischen Gelenkoperationen aller großen Gelenke, die aufgrund der Verletzungshäufigkeit unterschiedlich oft betroffen sind. Gelenke mit den häufigsten Eingriffen und den meisten Operationstechniken sind Knie und Schulter, gefolgt von oberem Sprunggelenk, Ellenbogen und Handgelenk. Für gelenknahe Frakturen wurden Implantate und Operationstechniken für minimalinvasive Plattenosteosynthesen (MIPO) und minimalinvasive perkutane Plattenosteosynthesen (MIPPO)

\section{Minimally invasive trauma surgery}

\section{Abstract}

Arthroscopic operations on the major joints account for a large proportion of those described as 'minimally invasive trauma surgery'. The frequency of such operations in the different joints varies with the frequency with which they are injured. The joints onwhich the most frequent arthroscopic procedures and the greatest number of different arthroscopic techniques are performed are the knee and the shoulder, followed in order by the ankle, the elbow and the wrist. Implants and surgical techniques have been developed to allow minimally invasive plate osteosynthesis (MIPO) and minimally invasive percutaneous plate osteosynthesis (MIPPO) in the treatment of entwickelt, hierdurch lassen sich die Ausheilungsergebnisse deutlich verbessern und Komplikationen drastisch reduzieren. Durch ständige Weiterentwicklung von arthroskopischen und minimalinvasiven Operationstechniken, Instrumenten und Implantaten wird Gelenk- und gelenknahe Chirurgie in naher Zukunft gleichzusetzen sein mit minimalinvasiver Unfallchirurgie.

\section{Schlüsselwörter}

Arthroskopie · Kniegelenk · Schulter · Ellenbogen · Sprunggelenk · Handgelenk

metaphyseal fractures. These techniques have made it possible to significantly improve final outcomes and to reduce the incidence of major complications substantially. Continuous development of arthroscopic and minimally invasive surgical techniques, instruments and implants means that in the near future the treatment of joint injuries and metaphyseal fractures will be synonymous with minimally invasive trauma surgery.

\section{Keywords}

Arthroscopy · Knee · Shoulder · Elbow · Ankle joint $\cdot$ Wrist 


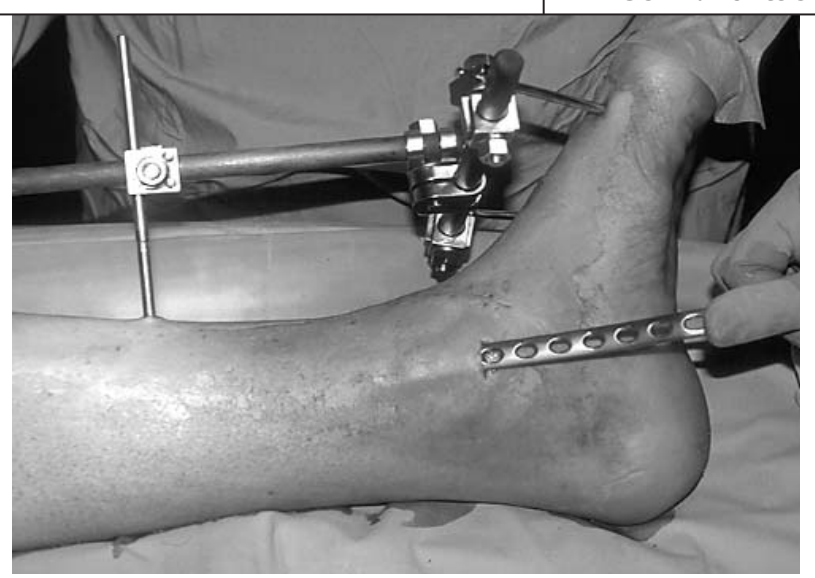

Abb. $2<$ Perkutane Plattenosteosynthese einer Pilon-tibiale-Fraktur
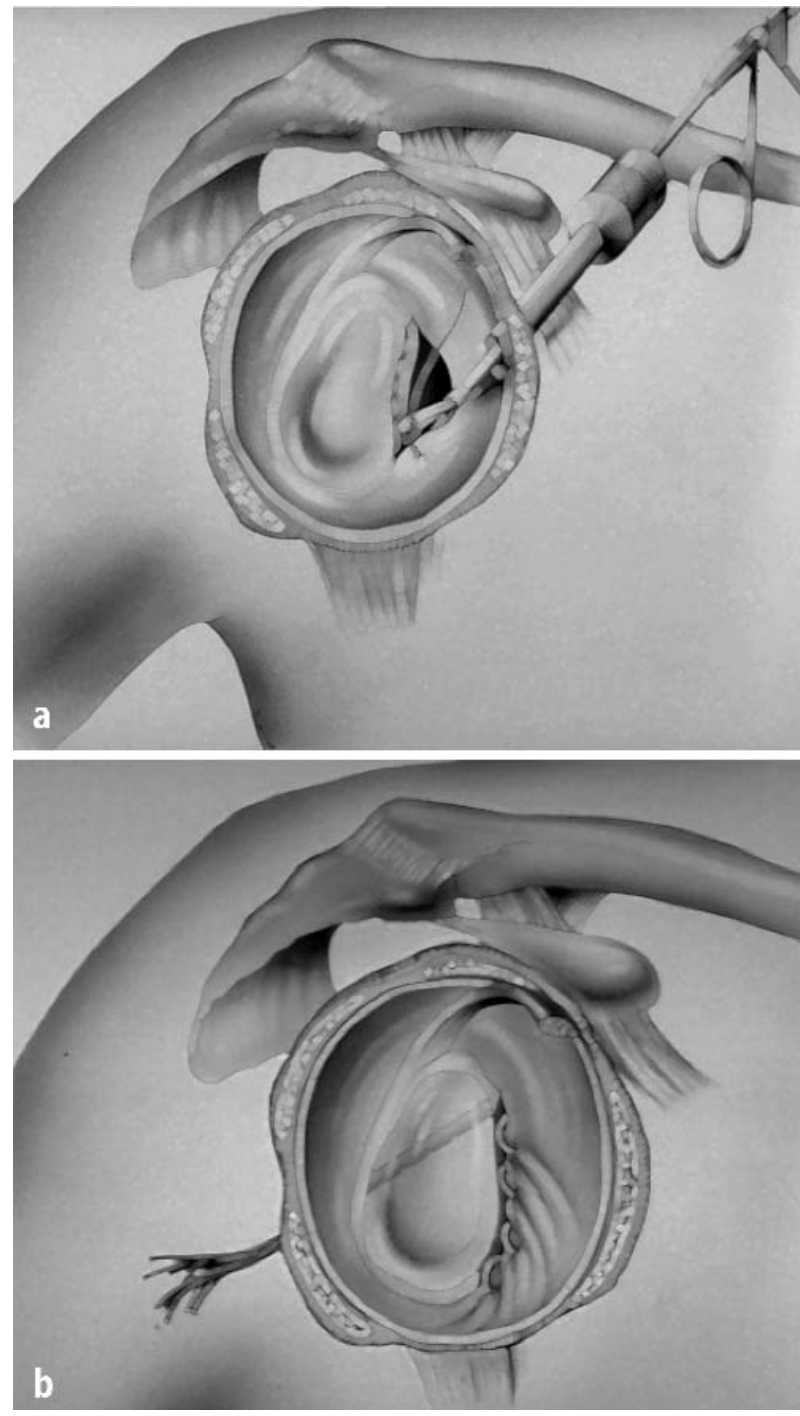

Abb. 3a, b $<$ Arthroskopische Schulterstabilisierung: a Nahtzange bei Platzierung von resorbierbaren Nähten im Bereich des verletzten Labrums, b schematische Darstellung der transglenoidal nach dorsal ausgeleiteten Fäden, nach Anspannen der Fäden legt sich das Labrum an korrekter Position wieder an

Thal [2], eine transglenoidale Nahttechnik (• Abb. 3).

Nach einer arthroskopischen Operation wird die operierte Schulter noch teilweise ruhig gestellt. Während des Tags ist der Arm in einer Orthese geführt, die die Bewegung in den nicht erlaubten Bewe- gungsrichtungen erschwert, während der Nacht wird der Arm in einem GilchristVerband ruhig gestellt. Für 4 Wochen darf nicht über $90^{\circ}$ abduziert oder antevertiert werden, die Außenrotation ist in dieser Zeit gänzlich verboten. Bereits am 1. postoperativen Tag beginnt die krankengym- nastische Übungsbehandlung, die diese Einschränkungen berücksichtigt. Nach Abschluss des Muskelaufbautrainings sollte der Trainingszustand bei aktiven Sportlern mit einem Messverfahren überprüft werden, bevor eine Wiederaufnahme sportlicher Aktivitäten erfolgt. Erst bei seitengleicher Muskelfunktion gestatten wir zu diesem Zeitpunkt, meist nach 36 Monaten, die volle Sportfähigkeit.

Hintere Instabilitäten und Läsionen des Bizepssehnenankers (SLAP-Läsionen) werden mit arthroskopischen Nahtverfahren versorgt, die Ruhigstellung und Nachbehandlung erfolgen jeweils an die Verletzung angepasst.

\section{Impingementsyndrome und Rotatorenmanschette}

Nach der Erstbeschreibung von Rissen der Rotatorenmanschette durch J.G. Smith 1834 werden eine Reihe von verschiedenen und $z$. T. sehr unterschiedlichen Ursachen als mögliche Auslöser für die pathologischen Veränderungen der Rotatorenmanschette angesehen, wobei in den meisten Fällen nicht eine einzelne, sondern eine Kombination von Ursachen für die Schädigung verantwortlich ist.

1972 beschrieb Neer [23] das Impingementsyndrom (- Tabelle 2), das er in die bekannten 3 Stadien einteilte. Gleichzeitig veröffentlichte er die Technik der offenen Akromioplastik als Therapie des Impingementsyndroms.

\section{Arthroskopische Techniken}

Impingementsyndrom. Bei manifesten Outlet-Impingementsyndromen erfolgt bei ausbleibender Besserung durch eine zunächst durchgeführte konservative Therapie eine arthroskopische subakromiale Dekompression. Dabei wird eine anterolaterale Resektion der meist entzündlich veränderten Bursa subacromialis über eine laterale Stichinzision vorgenommen. Anschließend wird über die gleiche Stichinzision mit einer 5,5 mm durchmessenden Kugelfräse der knöcherne Ansatz des Lig. coracoacromiale reseziert. $\mathrm{Zu}$ sätzlich werden das AC-Gelenk dargestellt und ggf. dort bestehende Osteophyten abgetragen. Letzter Arbeitsschritt ist die anterolaterale Akromioplastik, wobei sich die Resektionshöhe an der präoperativen 
Tabelle 1

\section{Einteilung der Schulterinstabilitäten nach Schneeberger et al. [27]}

$\begin{array}{ll}\text { Grad } & \text { Ausprägung } \\ \text { I } & \text { Chronisch verhakte Luxation } \\ \text { II } & \text { Unidirektionale Instabilität ohne Hyperlaxizität } \\ \text { III } & \text { Unidirektionale Instabilität mit multidirektionaler Hyperlaxizität } \\ \text { IV } & \text { Multidirektionale Instabilität ohne Hyperlaxizität } \\ \text { V } & \text { Multidirektionale Instabilität mit multidirektionaler Hyperlaxizität } \\ \text { VI } & \text { Willkürliche Instabilität }\end{array}$

Tabelle 2

Einteilung des Impingementsyndroms nach Neer [23]

\begin{tabular}{ll} 
Stadium & $\begin{array}{l}\text { Ausprägung } \\
\text { I }\end{array}$ \\
$\begin{array}{l}\text { Ödematöse Schwellung mit Einblutung in die Sehne, reversibles Krankheitsbild } \\
\text { des jungen Patienten }\end{array}$ \\
\hline II & Fibrosierung, Tendinitis und Verdickung von Sehne und Bursa subacromialis \\
\hline & $\begin{array}{l}\text { Chronischer, mechanisch-entzündlicher Reiz mit konsekutiver inkompletter } \\
\text { (akromialseitig, intratendinös, gelenkseitig) oder kompletter Ruptur der Sehne }\end{array}$ \\
\hline
\end{tabular}

Tabelle 3

Einteilung der Rotatorenmanschettenruptur nach Bateman [1]

Bezeichnung

$[\mathrm{cm}]$

Klein $\quad<1$

Mittel 1-3

Groß 3-5

Massiv $>5$

Röntgendiagnostik orientiert. Abschließend wird die bursaseitige Rotatorenmanschette beurteilt und ggf. débridiert.

Ab dem 1. postoperativen Tag erfolgt eine funktionelle Übungsbehandlung mit Fortführung der präoperativ eingeleiteten intensiven Krankengymnastik und Elektrotherapie.

Rotatorenmanschette. In Fällen von klinisch nachweisbaren Rotatorenmanschettenrupturen, bei denen eine freie Schulterfunktion mit einer mehr oder minder ausgeprägten Kraftminderung besteht und der Schulterschmerz das Leitsymptom darstellt, wurde und wird nach vorausgegangener konservativer Therapie häufig eine arthroskopische Dekompression empfohlen. Hier hat sich jedoch gezeigt, dass im direkten Vergleich zwischen alleiniger Dekompression und offener Rekonstruktion der Manschette Letztere $\mathrm{zu}$ besseren Resultaten führt. Weiterhin berichteten verschiedene $\mathrm{Au}$ - toren, dass im Langzeitverlauf mit einer weiteren Verschlechterung der anfänglich recht guten Ergebnisse nach alleiniger Dekompression gerechnet werden muss und in vielen Fällen dennoch eine Manschettenrekonstruktion erforderlich wird.

Andere Autoren propagierten eine frühzeitige Rekonstruktion der Rotatorenmanschette, da dadurch gleichzeitig die Schmerzsymptomatik beseitigt wird und die Ergebnisse deutlich besser sind als bei einer späteren Rekonstruktion. Für eine frühe Rekonstruktion sprechen zudem:

- Akute Rupturen haben eine bessere Prognose als chronische.

- Die Sehnen- bzw. Rupturränder retrahieren sich im Lauf der Zeit.

- Die Muskulatur der rupturierten Sehne degeneriert.

- Nach operativer Rekonstruktion ergeben sich gute bis sehr gute Ergebnisse

Bei kleineren Rupturen (Einteilung nach Bateman [1], • Tabelle 3) werden heute von einer Reihe von Autoren eine arthroskopisch assistierte Rekonstruktion bzw. eine arthroskopische Dekompression in Verbindung mit einer Mini-open-Rekonstruktion empfohlen, da die Ergebnisse der arthroskopischen bzw. der arthroskopisch assistierten Rekonstruktionen bei kleineren und mittleren Rotatorenmanschettenrupturen gleich gute Ergebnisse im Vergleich zur offenen Rekonstruktion zeigen.

Bei mittleren und großen Rupturen ist häufig ein offenes Vorgehen erforderlich, da die Manschette mobilisiert werden muss, damit ein spannungsfreier Verschluss gelingt. Die Technik des Defektbzw. Rupturverschlusses richtet sich nach der Lage und Größe des Defekts bzw. der Ruptur. Für alle ansatznahen Rupturen wird eine knöcherne Reinsertion im Bereich des ehemaligen Ansatzes am Tuberculum majus oder minus in eine mit einem Meißel hergestellte Knochennut durchgeführt, da dies eine optimale Revaskularisation der Sehne gewährleistet. Wie bei den arthroskopischen oder arthroskopisch assistierten Techniken wird auch bei offenem Vorgehen neben der Rekonstruktion der Manschette immer eine Dekompression bzw. Operation nach Neer [23] durchgeführt.

\section{Knieregion (Kniegelenk, distales Femur, proximale Tibia)}

\section{Vorderes Kreuzband}

Die Kreuzbandverletzung ist die häufigste Bandverletzung des Kniegelenks. In den USA wird anhand von Schätzungen eine Häufigkeit von 90.000 frischen vorderen Kreuzbandverletzungen pro Jahr angenommen. Das Verhältnis von Verletzungen des vorderen zum hinteren Kreuzband beträgt etwa 10:1. Bei zunehmender Beliebtheit des Breitensports in sämtlichen Altersgruppen werden die Diagnostik, die operative Therapie und die Rehabilitation der Kreuzbandverletzungen auch in Zukunft von großem klinischem Interesse bleiben.

Isolierte vordere Kreuzbandverletzungen sind meist Folge von Rotations-, Dezelerations- und/oder Hyperextensionsmechanismen. Die meisten Patienten können die Veränderung im Gelenkgefüge und die daraus resultierende Instabilität oft gut beschreiben. Ungefähr 40-65\% der Patienten verspüren einen Riss oder Knall zum Zeitpunkt der Verletzung. Die entstandene Instabilität sowie die sich rasch entwickelnde Schwellung aufgrund des Hämarthros machen 


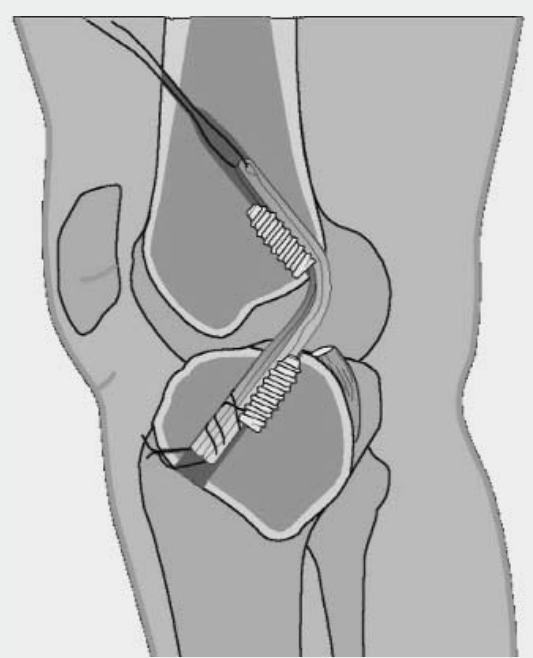

Abb. $4<$ Schematische Darstellung eines vorderen Kreuzbandersatzes mit einem 4Fach-Transplantat aus Semitendinosus-Gracilis-Sehne fixiert mit resorbierbaren Polylaktidinterferenzschrauben

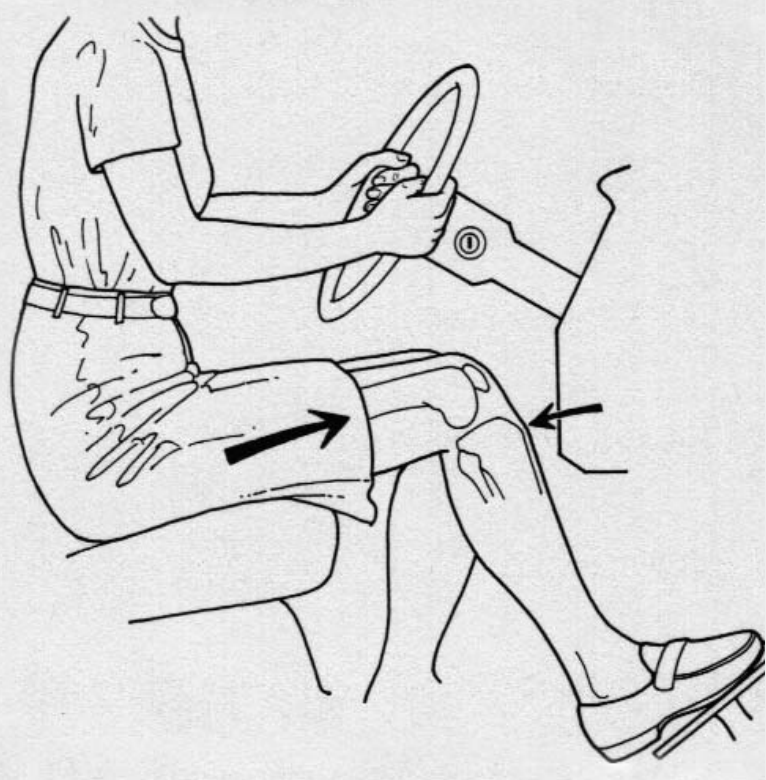

Abb. $5<$ Anpralltrauma am Armaturenbrett des Autos als Unfallursache für eine hintere Kreuzbandruptur eine Fortsetzung der sportlichen Aktivität unmöglich. Bis zum Beweis des Gegenteils sollte bei jedem Knieverletzten mit einer solchen oder ähnlich gearteten Anamnese als Verdachtsdiagnose eine Verletzung des vorderen Kreuzbands angenommen werden.

\section{Behandlung}

Wenn die Diagnose der vorderen Kreuzbandruptur gesichert ist, muss für den betroffenen Patienten der individuell abgestimmte optimierte Therapieplan erstellt werden.

Spontanverlauf. In den meisten Studien konnte gezeigt werden, dass die entstandene anteromediale Instabilität mit Ein- schränkungen mit bestimmten körperlichen Aktivitäten wie z. B. Hüpfen oder Drehbewegungen einhergeht. Ausheilungsergebnisse von 72 überwiegend männlichen Militärangehörigen mit einem Durchschnittsalter von 25 Jahren mit hohem körperlichem Aktivitätsniveau, die mit arthroskopisch gesicherter Ruptur des vorderen Kreuzbands einem standardisierten Rehabilitationsprogramm unterworfen wurden, zeigten im Rahmen der Nachuntersuchung nur $11 \%$ exzellente, $20 \%$ gute, $15 \%$ befriedigende und $54 \%$ schlechte funktionelle Ergebnisse. Insgesamt erreichten nur 5\% dieser Patienten ihren vorherigen Aktivitätslevel, bei 49\% wurde eine spätere Ersatzplastik des vor- deren Kreuzbands durchgeführt. Die Autoren kamen zu der Schlussfolgerung, dass die konservative Therapie bei jüngeren, körperlich aktiven Patienten mit überwiegend schlechten funktionellen Ausheilungsergebnissen behaftet ist.

Auch andere Studien zeigten ähnliche Ergebnisse, wohingegen Verlaufsuntersuchungen bei Patienten mit einer erfolgten Kreuzbandersatzplastik eine deutlich geringere Gelenkinstabilität und eine erheblich verminderte Inzidenz sekundärer Meniskusschäden aufwiesen. Es konnte weiter gezeigt werden, dass im konservativ behandelten Patientenkollektiv mit vorderer Kreuzbandinstabilität vermehrt Giving-way-Ereignisse auftraten, welche im weiterem Verlauf mit Meniskus- und Knorpelverletzungen einhergingen und in einigen Fällen einen vorzeitigen $\mathrm{Ge}$ lenkverschleiß zur Folge hatten.

Arthroskopische Techniken des Kreuzbandersatzes. In biomechanischen und klinischen Studien konnte mehrfach die wesentliche Bedeutung des vorderen Kreuzbands in der Aufrechterhaltung einer normalen Kniefunktion und Stabilität gezeigt werden. Dementsprechend wird bei der Rekonstruktion bzw. dem Ersatz der Berücksichtigung der normalen intraartikulären Anatomie eine wesentliche Bedeutung eingeräumt. Das bessere Verständnis der biomechanischen Anforderungen, die Fortschritte in der operativen Technik und eine zusätzliche Verbesserung der postoperativen Rehabilitation haben die Erfolgsrate erhöht und die postoperative Morbidität sowie die Komplikationsrate weiter reduziert.

Als Transplantate kommen überwiegend die Sehnen der Flexoren (M. semitendinosus, $M$. gracilis) sowie das mittlere Patellarsehnendrittel mit anhängenden Knochenblöcken (Bone-patella-boneTransplantat) zur Anwendung. Der Einsatz dieser Transplantate erfolgt heute überwiegend arthroskopisch (• Abb. 4).

Als Vorteile für ein arthroskopisches Vorgehen gelten der bessere Zugang und die bessere Einsicht in das Knie, der Verzicht auf größere Kapselinzisionen, das Arbeiten im flüssigen Medium (Vermeidung des Austrocknens von Weichteilen und Knorpel), die geringere Muskelatrophie im Vergleich zum offenen Vorgehen und die verminderte Inzidenz des 
postoperativen patellofemoralen Schmerzes im Vergleich zur offenen Rekonstruktion.

Der wesentliche Vorteil des arthroskopischen gegenüber einem operativen Vorgehen mit einer Miniarthrotomie, bei der lediglich der Hoffa-Körper gespalten wird, besteht in der im Vergleich beschleunigten Rehabilitation nach arthroskopischer Technik.

\section{Hinteres Kreuzband}

Verletzungen des hinteren Kreuzbands sowie der posterolateralen Kniegelenkstrukturen haben in den letzten Jahren zunehmend an Bedeutung gewonnen, wenngleich das Wissen ungleich geringer als das hinsichtlich des vorderen Kreuzbands ist. Von vielen Autoren wird das hintere Kreuzband als Hauptstabilisator des Kniegelenks angesehen. Trotz der eindeutigen anatomischen Bedeutung des hinteren Kreuzbands sind die Einschätzungen der Auswirkungen einer Ruptur desselben unterschiedlich. Das Beschwerdeausmaß einer hinteren Kreuzbandruptur weist eine erhebliche klinische Variationsbreite auf mit nur geringen funktionellen Beeinträchtigungen bei einem Großteil der Patienten.

In ungefähr der Hälfte der hinteren Kreuzbandverletzungen handelt es sich um isolierte Rupturen, wobei weitere Bandstrukturen nicht in Mitleidenschaft gezogen sind. Ein typischer Unfallmechanismus ist der Sturz auf das gebeugte Bein oder die Dashboard-Verletzung (• Abb. 5). Dementsprechend stellen Verkehrsunfälle die häufigste Ursache für hintere Kreuzbandrupturen dar.

Das Behandlungskonzept ist Gegenstand der aktuellen wissenschaftlichen Diskussion. Hierbei ist zum einem problematisch, dass größere Studien über den spontanen Langzeitverlauf nur in geringer Zahl vorhanden und die aktuellen Ergebnisse der operativen Behandlung oft sehr unterschiedlich sind. In verschiedenen Studien konnte aber gezeigt werden, dass nach einer Ruptur des hinteren Kreuzbands im weiteren Verlauf mit degenerativen Knorpelschäden, insbesondere im medialen Kompartment sowie im patellofemoralen Gelenk, zu rechnen ist. In den Fällen, in denen eine Operation in-

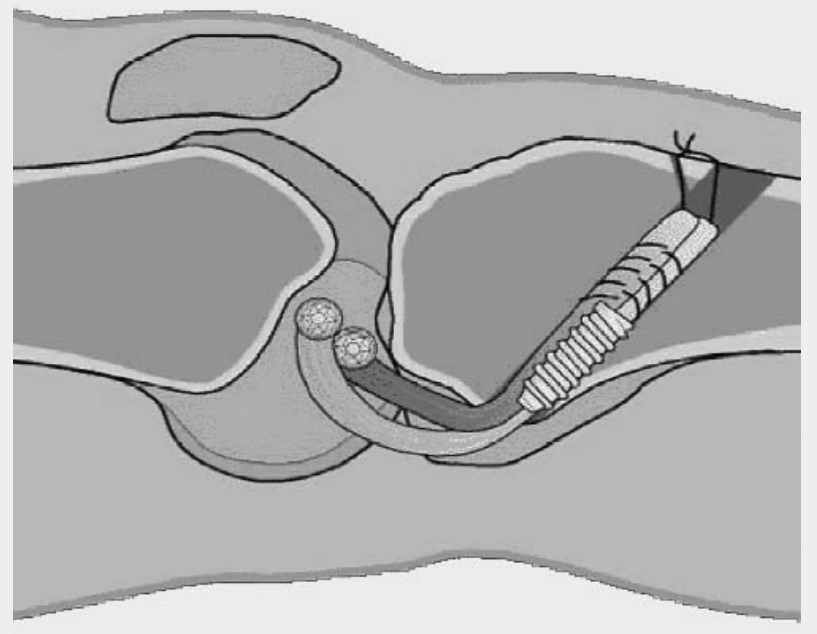

Abb. $6<$ Schematische Darstellung eines hinteren Kreuzbandersatzes mit einem Doppelbündeltransplantat aus Semitendinosus-GracilisSehne fixiert mit resorbierbaren Polylaktidinterferenzschrauben
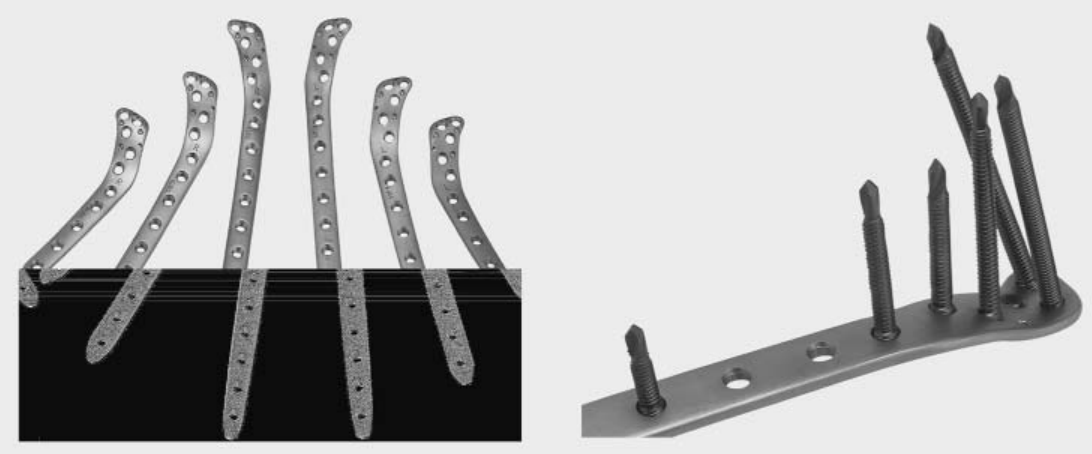

Abb. $7 \triangle$ Fixateur interne - LISS-PT: perkutanes Plattensystem für die proximale Tibia

diziert ist, wird sie in arthroskopischer Technik durchgeführt (• Abb. 6).

\section{Meniskus}

Anstelle der traditionellen Meniskektomie kommen heute, insbesondere durch die Möglichkeiten der Arthroskopie sowie der verfeinerten arthroskopischen Techniken, zunehmend schonende, sparsame Resektionsverfahren bzw. - wenn immer möglich - sogar Meniskusrefixationen zum Einsatz. Die Meniskusresektion beschränkt sich auf das notwendige Minimum, um weitere Rissbildungen zu vermeiden oder instabile Meniskusteile im geschädigten Bereich zu beseitigen. Wo immer möglich, wird eine Meniskus erhaltende Vorgehensweise angestrebt. Horizontale, parallel zum Meniskusrand verlaufende Risse, die in der roten Zone oder der rot-weißen Übergangszone liegen, werden refixiert.

\section{Allgemeine Prinzipien} der partiellen Meniskektomie

Die arthroskopische Operationstechnik bei der Meniskusresektion ist heute das Standardverfahren. Sie ermöglicht durch den besseren Einblick in den Hinterhornbereich im Vergleich zum früher üblichen offenen Vorgehen die partielle Meniskektomie. Das Ziel ist, durch bogenförmige Resektion einen glatten, stabilen Resektionsrand zu erreichen, wobei im Restmeniskus keine Rissbildung mehr vorhanden sein darf. Der neu gebildete freie Rand sollte auch keine Ecken aufweisen.

Voraussetzung für die operative Versorgung von Meniskusverletzungen ist eine genaue Erfassung der Art und des Ausmaßes der Rissbildung. Dazu ist zunächst eine ausreichende Sicht auf den Meniskus notwendig. Aber die alleinige Inspektion gibt nicht genügend Information über das Schadensausmaß; erst die Palpation der Rissbildung, das Bewegen 
verletzter oder teilweiser losgelöster Meniskusanteile und das Anheben des Meniskus zur Inspektion auch der Unterfläche ermöglichen die exakte Erfassung des Schadens. Die häufigsten Rissbildungen sind in $\bullet$ Abb. 7 dargestellt.

Totale Meniskektomien sind heutzutage die absolute Ausnahme. Durch die Möglichkeiten der operativen Arthroskopie sind sehr sparsame Resektionen möglich geworden. Mit die Weiterentwicklung von arthroskopischen Instrumenten können Meniskusrisse jeweils so behandelt werden, dass die bestmögliche Erhaltung gewährleistet ist. Darüber hinaus besteht in geeigneten Fällen die Möglichkeit der Meniskusrefixation.

\section{Tibiakopffrakturen}

Die Arthroskopie stellt eine wertvolle diagnostische Ergänzung in den Fällen dar, in denen Zweifel über das Ausmaß der Gelenkbeteiligung und der Gelenkstufen bestehen. Sie kann im Rahmen der operativen Frakturversorgung als diagnostische Arthroskopie vorangestellt werden.

Eine weitere Einsatzmöglichkeit besteht heute in der arthroskopisch und mit Bildwandler unterstützten minimalinvasiven Chirurgie von Tibiakopfbrüchen. Hierfür eignen sich aber prinzipiell nur die Plateaufrakturen. Ein Vorteil dieser Vorgehensweise sind die arthroskopische Diagnostik und Therapiemöglichkeit von Begleitverletzungen der Menisken und Bänder, die auch bei Plateaufrakturen in einem nicht unerheblichen Anteil auftreten können.

Die minimalinvasive, arthroskopische Technik umfasst nach der arthroskopischen Diagnostik und der Beurteilung von Begleitverletzungen die Reposition und perkutane Stabilisierung der Fraktur. Erstere erfolgt über ein kleines vorderes Knochenfenster unterhalb der Frakturregion. Über dieses werden die knöchernen Strukturen des imprimierten Frakturabschnitts hochgestößelt, wobei dieses Repositionsmanöver arthroskopisch und radiologisch kontrolliert wird. Bei zufrieden stellendem Repositionsergebnis erfolgt zunächst die temporäre Fixation mittels perkutan eingebrachter KirschnerDrähte, danach die endgültige Stabilisierung mit kanülierten Spongiosaschrau- ben. Verbleibende größere metaphysäre Knochendefekte unter den angehobenen Fragmenten werden z. B. mit einer Hydroxylapatitkeramik (Endobon) oder autogener Spongiosa aufgefüllt.

Bei Frakturen, die sich metaphysär weiter nach distal erstrecken, wird der Gelenkanteil der Fraktur arthroskopisch kontrolliert durch eine kanülierte Verschraubung versorgt, die zusätzlich erforderliche Stabilisierung zum Tibiaschaft wird mit einem perkutan eingebrachten Fixateur interne, der LISS-PT (• Abb. 7), durchgeführt.

\section{Fazit}

Goldstandard in der Gelenk- und gelenknahen Chirurgie sind bereits vielfach arthroskopische Operationstechniken, die auch aufgrund kürzerer Behandlungsdauer und besserer kosmetischer Ergebnisse vorzuziehen sind.

Durch ständige Weiterentwicklung von arthroskopischen und minimalinvasiven Operationstechniken, Instrumenten und Implantaten wird Gelenk- und gelenknahe Chirurgie in naher Zukunft mit minimalinvasiver Unfallchirurgie gleichzusetzen sein.

\section{Korrespondierender Autor Prof.Dr. N.P. Südkamp}

Department Orthopädie und Traumatologie, Klinik für Traumatologie, Universitätsklinikum, Hugstetter Straße 55,79106 Freiburg E-mail: suedkamp@ukl.uni-freiburg.de

Interessenkonflikt: Der korrespondierende Autor versichert, dass keine Verbindungen mit einer Firma, deren Produkt in dem Artikel genannt ist, oder einer Firma, die ein Konkurrenzprodukt vertreibt, bestehen.

\section{Literatur}

1. Bateman JE (1983) Neurologic painful conditions affecting the shoulder. Clin Orthop 173: 44-54

2. Caspari RB, Thal R (1992) A technique for arthroscopic subacromial decompression. Arthroscopy 8: 23-30

3. Fanelli GC (1997) Posterior cruciate ligament 1997: indications for reconstruction, techniques, results. Instructional Course, Arthroscopy Association North America, Rosemont, IL

4. Feagin JA (ed) (1995) The crucial ligaments: diagnosis and treatment of ligamentous injuries about the knee. Churchill Livingstone, Edinburgh London New York

5. Fowble CD, Zimmer JW, Schepsis A (1993) The role of arthroscopy in the assessment and treatment of tibia plateau fractures. Arthroscopy 9: 584-590

6. Fu FH, Woo SL, Irrgang JL (1993) Current concepts for rehabilitation following anterior cruciate ligament re construction. J Orthop Sports Phys Ther 15: 270-278
7. Glashow JL, Friedman MJ (1991) Diagnosis of knee ligament injuries: magnetic resonance imaging. In: Scott WN (ed) Ligament and extensor mechanism injuries of the knee: diagnosis and treatment. Mosby Year Book, St Louis

8. Goldberg VM (1991) Controversies of total knee arthroplasty. Raven Press, New York

9. Guanche CA, Markman AW (1993) Arthroscopic management of tibial plateau fractures. Arthroscopy 9: 467-471

10. Harner CD (1995) The human posterior cruciate ligament complex: an interdisciplinary study - ligament morphology and biomechanical evaluation. Am J Sports Med 23: 736

11. Harner CD (1996) The posterior cruciate overview. Instructional Course, American Academy of Orthopaedic Surgeons, Rosemont, IL

12. Irrgang JL (1993) Modern trends in the anterior cruciate ligament rehabilitation: nonoperative and postoperative management. Clin Sports Med 12: 797-812

13. Itokazu M, Matsunaga T (1993) Arthroscopic restoration of depressed tibial plateau fractures using bone and hydroxyapatite grafts. Arthroscopy 9: 103-108

14. Jackson D (ed) (1993) The anterior cruciate ligament. Raven Press, New York

15. Jakob RP, Stäubli HU (ed) (1990) Kniegelenk und Kreuzbänder.Springer, Berlin Heidelberg New York

16. Kennedy JC, Grainger RW (1967) The posterior cruciate ligament. J Trauma 7: 367-377

17. LaPrade RF, Terry GC (1997) Injuries to the posterolateral aspect of the knee - association of anatomic injury patterns with clinical instability. Am J Sports Med 25: 433

18. Lobenhoffer P, Lattermann C, Krettek C et al. (1996) Rupturen des hinteren Kreuzbandes: Heutiger Behandlungsstand. Unfallchirurg 99: 382

19. Manal TJ, Snyder-Mackler $L$ (1996) Practice guidelines for anterior cruciate ligament rehabilitation: a criterionbased rehabilitation progression. In: Balderston RA, Bartoluzzi AR (eds) Operative techniques in orthopaedics, vol 6: Surgery of the anterior cruciate ligament. Mosby, St Louis, pp 190-196

20. Mont MA, Scott WN (1991) Classification of ligament injuries. In: Scott WN (ed) Ligament and extensor mechanism injuries of the knee: diagnosis and treatment. Mosby Year Book, Chicago

21. Mow VC (1992) Knee meniscus: basic science and clinical foundations. Raven Press, New York

22. Müller W (Hrsg) (1982) Das Knie. Springer, Berlin Heidelberg New York

23. Neer CS 2nd (1972) Anterior acromioplasty for the chronic impingement syndrome in the shoulder: a preliminary report.J Bone Joint Surg Am 54: 41-50

24. O'Dwyer KJ, Bobic VR (1992) Arthroscopic management of tibia plateau fractures. Injury 23: 261-264

25. Paulos LE, Payne FC, Rosenberg TD (1987) Rehabilitation after anterior cruciate ligament surgery. In: Jackson DW, Drez D (eds) The anterior cruciate deficient knee. Mosby, St Louis, pp 291-314

26. Reeves R (1966) Arthrography of the shoulder. J Bone Joint Surg Br 48: 424-435

27. Schneeberger AG, Hersche 0, Gerber C (1997) [The unstable shoulder.Classification and therapy]. Orthopäde 26: 909-914

28. Shelbourne KD, Klootwyk TE, DeCarlo F (1992) Update on accelerated rehabilitation after anterior cruciate ligament reconstruction.J Orthop Sports Phys Ther 15: 303-308

29. Stähelin AC, Südkamp NP, Weiler A (2001) Anatomic double-bundle posterior cruciate ligament reconstruction using hamstring tendons. Arthroscopy 17: 88-97

30. Strobel M, Eichhorn J, Schießler W (Hrsg) (1998) Arthroskopie des Kniegelenks - Grundprinzipien, diagnostische Arthroskopie, arthroskopische Chirurgie, 3. Aufl. Deutscher Ärzteverlag, Köln 
31. Südkamp NP (2001) Die Rotatorenmanschettenruptur. Zentralbl Chir 126: 1-7

32. Südkamp NP, Haas NP (2000) Neue Wege in der Kreuzbandchirurgie. Chirurg 71: 1024-1033

33. Südkamp N, Lobenhoffer P, Haas N et al. (1994) Arthroscopic capsulorrhaphy for anterior shoulder instability (Caspari procedure). Orthop Traumatol 3: 1-10

34. Südkamp N, Granrath M, Hoffmann R et al. (1994) Instabilitäten des Schultergelenkes beim Sportler.Chirurg 65: 901-909

35. Südkamp N, Schönfelder V, Weiler A et al. (1998) Arthroskopisch kontrollierte minimal invasive Stabilisierung von Tibiakopffrakturen. Arthroskopie 11: 228-234

36. Sweitzer RW, Sweitzer DA, Sarniti AJ (1991) Rehabilitation for ligament and extensor mechanism injuries. In: Scott WN (ed) Ligament and extensor mechanism injuries of the knee: diagnosis and treatment. Mosby Year Book, Chicago

37. Thomas SC, Matsen FA 3rd (1989) An approach to the repair of avulsion of the glenohumeral ligaments in the management of traumatic anterior glenohumeral instability.J Bone Joint Surg Am 71: 506-513
„Trauma und Berufskrankheit" bietet aktuelle Beiträge zu Fragen der Unfall- und Wiederherstellungschirurgie, der Berufserkrankungen sowie zu Themen der Unfallverhütung, der operativen und konservativen Therapie.

Möchten Sie ein bereits erschienenes Heft nachbestellen? Die folgenden Ausgaben können Sie direkt bei unserem Kundenservice zum Preis von je EUR 35,- beziehen:

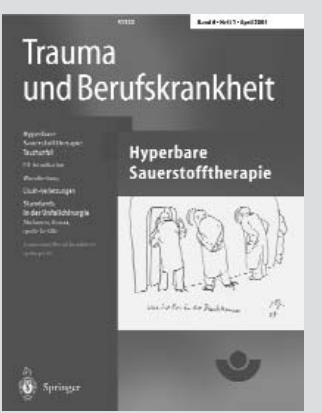

\section{Heft 1/2004}

Leitthema:

- Hyperbare Sauerstofftherapie

Standards in der Unfallchirurgie:

- Abdomen, Thorax, große Gefäße

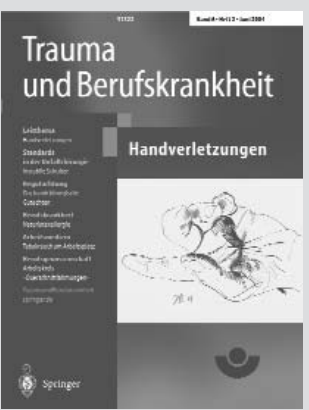

\section{Heft 2/2004}

Leitthema:

- Handverletzungen

Standards in der Unfallchirurgie:

- Instabile Schulter

Begutachtung:

- Das handchirurgische Gutachten

Berufskrankheit:

- Naturlatexallergie

Arbeitsmedizin:

- Tabakrauch am Arbeitsplatz

Begutachtung:

- Arbeitskreis „,Querschnittlähmungen“

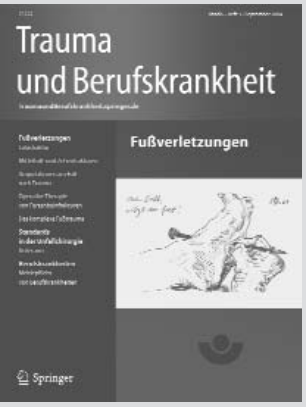

Heft 3/2004

Leitthema:

- Fußverletzungen

Standards in der Unfallchirurgie:

- Unterarm

Berufskrankheiten:

- Meldepflicht von Berufskrankheiten

So erreichen Sie unseren Kundenservice:

Springer-Verlag

Kundenservice Zeitschriften

Haberstr. 7,69126 Heidelberg

Tel.: +49 6221 345-4303

Fax: +49 $6221345-4229$

E-Mail:SAG-journals@springer-sbm.com

TraumaundBerufskrankheit.springer.de 